\title{
Pandemia da COVID-19: insatisfação com o trabalho entre professores(as) do estado de Minas Gerais, Brasil
}

\author{
COVID-19 pandemic: dissatisfaction with work \\ among teachers in the state of Minas Gerais, Brazil
}

Rosângela Ramos Veloso Silva (http://orcid.org/0000-0003-3329-8133) ${ }^{1}$
Rose Elizabeth Cabral Barbosa (http://orcid.org/0000-0001-5383-0102) ${ }^{1}$
Nayra Suze Souza e Silva (http://orcid.org/0000-0002-8420-0821) ${ }^{1}$
Lucinéia de Pinho (https://orcid.org/0000-0002-2947-5806) ${ }^{1}$
Thalita Bahia Ferreira (https://orcid.org/0000-0001-6764-3463) ${ }^{1}$
Bethânia Borja Moreira (https://orcid.org/0000-0002-6851-6631) ${ }^{1}$
Maria Fernanda Santos Figueiredo Brito (https://orcid.org/0000-0001-5395-9491) ${ }^{1}$
Desirée Sant'Ana Haikal (https://orcid.org/0000-0002-0331-0747) ${ }^{1}$

${ }^{1}$ Universidade Estadual de Montes Claros. Av. Prof. Rui Braga s/n, Vila Mauriceia. 39401-089 Montes Claros MG Brasil. rosangela.veloso@ unimontes.br

\begin{abstract}
This article aimed to verify the prevalence and factors associated with dissatisfaction with teaching work among teachers from the state public basic education network in the state of Minas Gerais during the COVID-19 pandemic. This is a websurvey, carried out with these teachers between August and September 2020 via digital form. The dependent variable was job satisfaction during the pandemic, with satisfied people being the reference category. Multinomial Logistic Regression was used. 15,641 teachers from $795 \mathrm{mu}$ nicipalities participated in the study. Regarding work satisfaction, $21.6 \%$ were satisfied, $44.7 \%$ were indifferent and $33.7 \%$ were unsatisfied. The chances of being unsatisfied were higher among those without a spouse $(O R=1.23)$, longer teaching time $(O R=1.19)$, difficulty with remote activities $(O R=37.60)$, without possession of a computer $(O R=1.40)$, smokers $(O R=1.27)$, using alcoholic beverages $(O R=1.54)$, sedentary $(O R=1.22)$ and absent leisure activities $(O R=1.49)$. The changes caused in the educational system in the face of the pandemic impacted the teacher's routine, contributing to the dissatisfaction with the work of this professional.
\end{abstract}

Key words Faculty, Job satisfaction, Health surveys, Pandemics, COVID-19
Resumo O objetivo deste artigo foi verificar a prevalência e fatores associados à insatisfação com o trabalho docente entre professores(as) da rede pública estadual de educação básica do estado de Minas Gerais durante a pandemia da COVID-19. Inquérito epidemiológico do tipo websurveys, realizado com esses(as) professores(as) entre agosto e setembro de 2020 via formulário digital. A variável dependente foi a satisfação com o trabalho durante a pandemia, sendo os(as) satisfeitos(as) a categoria de referência. Foi utilizada a Regressão Logistica Multinomial. Participaram do estudo 15.641 professores(as) de 795 municípios. Em relação a satisfação com o trabalho, 21,6\% estavam satisfeitos(as), 44,7\% indiferentes e $33,7 \%$ insatisfeitos(as). As chances de estarem insatisfeitos(as) foram maiores entre aqueles(as) sem cônjuge $(\mathrm{OR}=1,23)$, maior tempo de docência $(\mathrm{OR}=1,19)$, dificuldade com atividades remotas $(O R=37,60)$, sem posse de computador $(O R=1,40)$, tabagistas $(O R=1,27)$, em uso de bebida alcoólica $(O R=1,54)$, sedentários(as) $(O R=1,22)$ e atividade de lazer ausente $(O R=1,49)$. As mudanças causadas no sistema educacional diante da pandemia impactaram a rotina do(a) professor(a), contribuindo para a insatisfação com o trabalho deste(a) profissional.

Palavras-chave Professores, Satisfação no trabalho, Inquérito epidemiológico, Pandemia, COVID-19 


\section{Introdução}

A pandemia da COVID-19 e as medidas de prevenção à disseminação da doença implementadas ao redor do mundo representaram desafio expressivo aos mais diversos setores da sociedade. Em especial, o setor educacional foi enormemente afetado. Escolas públicas e privadas das diferentes etapas de ensino foram fechadas e as atividades ora desenvolvidas presencialmente ganharam formato virtual.

Em Minas Gerais, o governo do estado determinou o fechamento das escolas estaduais e a suspensão das aulas, por tempo indeterminado, a partir de 20 de março de $2020^{1}$. As atividades de ensino foram retomadas em 18 de maio por meio do regime de estudo não presencial, criado pela Secretaria de Estado de Educação que contava com a disponibilização dos Planos de Estudo Tutorados (PET), aplicativo específico e um programa de TV, além da utilização das redes sociais e do próprio site da Secretaria no intuito de integrar as ações propostas ${ }^{2}$.

Portanto, os(as) professores(as) se viram diante de aulas presenciais suspensas sem, contudo, que isso significasse a interrupção do processo de ensino-aprendizagem. A necessidade do distanciamento social e a implementação do ensino remoto tornou imprescindível, ainda que sem conhecimento ou familiaridade suficientes, o uso de ferramentas digitais de informação e comunicação ${ }^{3,4}$. A adoção dessa modalidade de ensino exigiu dos(as) professores(as) a adequação do ambiente domiciliar e a reestruturação dos planejamentos de ensino em um contexto de "disponibilidade irrestrita" 3 de tempo para responder às demandas de estudantes e familiares ${ }^{3,4}$.

Longe de um cenário ideal para o desenvolvimento do ensino remoto, os(as) professores(as) enfrentam diferentes desafios. Estudo realizado entre professores(as) de escolas primárias na Indonésia, durante a pandemia, identificou quatro grupos de desafios enfrentados durante o ensino online, incluindo: os obstáculos tecnológicos - a necessidade de possuir celular ou computador com acesso à internet -, as interferências de familiares durante as aulas, a dificuldade em manter a concentração e a participação dos estudantes e, por fim, a experiência dos(as) professores(as) em relação às ferramentas tecnológicas e a capacidade de compreensão de como elas funcionam ${ }^{5}$.

Há, ainda, a dificuldade das escolas em orientar os(as) professores(as) quanto ao uso de estratégias capazes de mediar a realidade do ensino remoto para dentro das casas dos(as) estudantes, delegando ao(à) professor(a) parte da responsabilidade em lidar com a evasão durante o ensino remoto e a necessidade que a aprendizagem continue a atingir as metas anteriormente estabelecidas $^{3,5}$. A responsabilização dos(as) professores(as) durante a pandemia associada à ausência de capacitações podem contribuir para a sobrecarga de trabalho, aumentando a exaustão, o que pode influenciar a satisfação com o trabalho que eles(as) desempenham ${ }^{3,6}$.

Investigações sobre a satisfação com o trabalho reafirmam o papel fundamental dos aspectos psicossociais do trabalho e consideram que o sentimento de satisfação está atrelado à interação entre condições de vida e trabalho. Dessa forma, tanto a satisfação com o trabalho é positiva para a saúde, quanto a insatisfação pode trazer prejuízos à saúde do trabalhador e à organização na qual ele está inserido ${ }^{7}$. Em tempos de trabalho presencial, sabe-se que o estresse causado pelo acúmulo de múltiplas tarefas, sobrecarga, desvalorização profissional e baixos salários dos(as) professores(as) pode desencadear um processo de permanente insatisfação, com sentimentos de fracasso e indiferença, levando ao adoecimento físico e mental e, ainda, ao desejo de abandonar a atividade docente ${ }^{6,8}$.

Diante disso, entender os fatores que estão associados a insatisfação como o trabalho docente pode contribuir para implementação de medidas que minimizem este efeito. Para tal, o presente estudo teve como objetivo verificar a prevalência e fatores associados a insatisfação com o trabalho docente entre professores(as) da rede pública estadual de educação básica de Minas Gerais durante a pandemia da COVID-19.

\section{Métodos}

Este estudo faz parte do Projeto ProfSMoc - Etapa Minas Covid "Condições de saúde e trabalho entre professores da rede estadual de ensino do estado de Minas Gerais na pandemia da COVID-19". Trata-se de um inquérito epidemiológico do tipo websurveys, realizado com professores(as) da educação básica (educação infantil, ensino fundamental e médio) das escolas da rede pública estadual de ensino de Minas Gerais, Brasil. A rede estadual de educação de Minas Gerais é composta por aproximadamente de 90.000 professores(as) (dado fornecido pela Secretaria de Estado de Educação de Minas Gerais - SEE-MG mediante folha de pagamento do mês de julho de 2020), atuantes em 3.441 escolas públicas es- 
taduais ${ }^{9}$. Por se tratar de websurvey, na tentativa de aumentar a qualidade e interpretação dos resultados obtidos ${ }^{10}$, o presente estudo seguiu as considerações do Checklist for Reporting Results of Internet E-Surveys (CHERRIES) ${ }^{11}$.

Para o cálculo amostral foi utilizada fórmula considerando populações infinitas. Foi considerada prevalência de $50 \%$ com a intenção de obter o maior tamanho amostral e consequentemente maior poder de inferência para diferentes variáveis. O erro tolerável adotado foi de 3\%. Além disso, a amostra foi duplicada (deff $=2)$, visto que a coleta foi proveniente de conglomerados. Também foi realizado acréscimo de $20 \%$ no tamanho amostral para compensar possíveis perdas (taxa de não resposta) que poderiam comprometer a validade do estudo. Assim, estimou-se a necessidade de se coletar dados de 2.564 professores(as) de escolas estaduais do estado de Minas Gerais para garantir a representatividade para todo estado.

Administrativamente, a SEE-MG divide o estado em seis polos e cada polo é subdividido em Superintendências Regionais de Ensino (SREs). Ao todo, o estado apresenta 45 SREs. $O$ acesso ao quantitativo de professores(as) e à qual SRE o(a) professor(a) estava vinculado(a) foi disponibilizada pela SEE-MG. Assim, a amostra foi estimada garantindo proporcionalidade dos(as) professores(as) por SRE, estimando o número mínimo de professores(as) a serem investigados(as) em cada uma delas.

Estudo piloto prévio foi realizado com 20 professores(as) de cinco diferentes cidades mineiras para teste, acerto do instrumento de coleta de dados e estimativa do tempo necessário para o preenchimento. Antes de iniciar a coleta de dados foram obtidas autorizações por parte da SEE -MG e das 45 SREs. A pesquisa foi divulgada nas mídias sociais da SEE-MG, sensibilizando para a participação dos(as) professores(as) na pesquisa. A coleta de dados ocorreu de 20 de agosto a 11 de setembro de 2020 por meio de formulário digital disponibilizado via plataforma Google Forms ${ }^{\circledR}$. O link do formulário foi enviado pela SEE-MG para o e-mail institucional de todos(as) os(as) professores(as) do estado, caracterizando uma coleta de dados "fechada" 11 aos(as) professores(as) da rede pública estadual. Para evitar o preenchimento automático do formulário por sistemas computacionais, foi utilizado um reCAPTCHA, que apresentava testes em imagens, impedindo que o formulário fosse enviado com sucesso por algum robô. O formulário continha 144 questões, dividido em 4 seções: características sociodemográficas, condição de trabalho, saúde e estilo de vida. Para algumas questões foram questionados a situação antes e durante a pandemia. No geral, o formulário foi baseado na pesquisa "ConVid - Pesquisa de Comportamentos”, realizada pela Fundação Oswaldo Cruz e parceiros ${ }^{12}$, e também incorporou outros instrumentos validados. Todas as questões do formulário foram de preenchimento obrigatório, minimizando perdas de informação. Os(as) participantes(as) tiveram opção de rever suas respostas a todo momento antes do envio do formulário. O estudo também garantiu o anonimato dos(as) professores(as) e o formulário de coleta de dados consumia aproximadamente de 25 minutos para ser preenchido.

Foram incluídos no estudo os(as) professores(as) em exercício da função docente no ano de 2020, os(as) atuantes na educação infantil, ensino fundamental e/ou ensino médio (vinculados(as) a alguma escola pública estadual de Minas Gerais) e aqueles(as) que aceitassem participar da pesquisa. Não participaram os(as) professores(as) que estivessem atuando em cargo diferente da função docente no contexto escolar (diretores(as), coordenadores(as), entre outros), os(as) aposentados(as) e aqueles(as) que responderam "não" quando perguntados(as) se aceitavam participar do estudo. Não houve restrição de participação aos(as) que estivessem afastados por licença médica.

A satisfação com o trabalho durante a pandemia foi a variável dependente adotada ("Durante o isolamento social devido a pandemia do novo coronavírus, como você se sente em relação a seu trabalho como docente?"). As opções de respostas seguiram escala Likert com três opções: "satisfeito(a)", "nem satisfeito(a) nem insatisfeito(a)" e "insatisfeito(a)". Para a categoria "nem satisfeito(a) nem insatisfeito(a)" adotou-se o termo "indiferente". As variáveis independentes foram organizadas em três blocos temáticos, listados a seguir.

1. Perfil sociodemográfico e econômico: sexo, idade, área censitária (referente a localização da escola estadual em que o professor trabalhava), renda familiar antes da pandemia (considerando o salário mínimo vigente de $\mathrm{R} \$ 1.045,00$ à época da coleta de dados), renda familiar durante a pandemia, outro trabalho remunerado, situação conjugal e filho(s).

2. Perfil ocupacional: anos de docência, horas semanais de trabalho docente, tipo de vínculo com a escola, se possui pós-graduação, realização de trabalho remoto durante a pandemia, dificuldades com atividades remotas e se possui computador. 
3. Comportamentos/Hábitos durante a pandemia: tabagismo, consumo de bebida alcóolica, prática de atividade física e atividade de lazer.

Os dados foram organizados, auditados e analisados com o auxílio do programa Statistical Package for Social Sciences (SPSS ${ }^{\circledR}$ ) versão 22.0. Foi conduzida estatística descritiva dos dados (frequência simples e relativa). Para a análise dos fatores associados à satisfação com o trabalho durante a pandemia, foram realizadas previamente análises bivariadas, utilizando o teste de Qui-quadrado de Pearson. Nessa análise, as variáveis que revelaram nível descritivo inferior ou igual a $20 \%(\mathrm{p} \leq 0,20)$ foram selecionadas para compor inicialmente o modelo múltiplo. No modelo múltiplo, adotou-se a Regressão Logística Multinomial, considerando a satisfação com o trabalho durante a pandemia como a categoria de referência. Os modelos foram manualmente ajustados. Todas variáveis que apresentaram $\mathrm{p} \leq 0,20$ entraram juntas no modelo, sendo retirada uma por uma, mantendo no modelo final apenas as variáveis que apresentaram nível descritivo inferior a $5 \%(\mathrm{p}<0,05)$. Foram estimadas a Odds Ratio (OR), o intervalo de $95 \%$ de confiança e nível descritivo. A qualidade do ajuste do modelo foi avaliada pelo coeficiente de determinação (Pseudo R²).

O Projeto ProfSMoc - Etapa Minas Covid foi submetido e aprovado pelo Comitê de Ética em Pesquisa da Universidade Estadual de Montes Claros (Unimontes). Todos(as) os(as) professores(as) participantes receberam junto ao formulário de coleta de dados o Termo de Consentimento Livre e Esclarecido informando sobre a metodologia do estudo, seus objetivos e confiabilidade das informações e assinalaram "sim" à questão relativa à concordância em participar da pesquisa. A pesquisa também cumpriu com a Resolução no 466/2012 do Conselho Nacional da Saúde/Ministério da Saúde, que trata de pesquisa com seres humanos.

\section{Resultados}

O formulário foi acessado por 16.210 professores(as), destes(as) 15.641 aceitaram participar da pesquisa, resultando em uma taxa de recrutamento $^{11}$ de $96,5 \%$ e taxa de completude ${ }^{11}$ de $100 \%$. Houve participação de professores(as) de 795 cidades mineiras $(93,2 \%)$. Entre eles(as), $81,9 \%$ eram do sexo feminino, $97,4 \%$ tinham até 60 anos e 59,5\% tinham renda familiar entre três e cinco salários mínimos. Quanto ao perfil ocu- pacional, $62 \%$ relataram mais de 10 anos de atuação docente e $54 \%$ eram concursados(as)/efetivos(as). Em relação ao comportamento/hábitos de vida durante a pandemia, $40,6 \%$ consumiam bebidas alcoólicas e $43,8 \%$ não estavam praticando atividade física (Tabela 1).

Em relação à satisfação com o trabalho durante a pandemia, $21,6 \%$ (3.375) dos(as) professores(as) estavam satisfeitos(as), 44,7\% (6.995) indiferentes e $33,7 \%$ (5.271) insatisfeitos(as). A prevalência de insatisfação com o trabalho foi maior entre: os homens, aqueles com idade de 21 a 40 anos, que atuavam na docência havia 21 anos ou mais e os que possuíam jornada de trabalho superior a 40 horas semanais. Demais dados da análise bivariada estão descritos na Tabela 1. Todas as variáveis independentes estiveram associadas à satisfação com o trabalho no nível de significância de $20 \%$.

Durante modelagem múltipla, as variáveis: filhos, horas semanais de trabalho docente e realização de trabalho remoto durante a pandemia foram removidas por apresentar $\mathrm{p}$-valor maior que 5\%. A Tabela 2 apresenta as variáveis que permaneceram no modelo múltiplo final após ajustes.

As chances de estarem indiferentes (quando comparados(as) aos(as) satisfeitos(as)) foram maiores nos(as) professores(as) que lecionavam na zona rural $(\mathrm{OR}=1,18)$, nos(as) que tiveram diminuição na renda familiar durante a pandemia $(\mathrm{OR}=1,41)$, nos(as) que viviam sem cônjuge $(\mathrm{OR}=1,13), \operatorname{nos}(\mathrm{as})$ que tinham entre 11 e 20 anos de docência $(\mathrm{OR}=1,16)$ e 21 anos ou mais de docência $(\mathrm{OR}=1,28)$, nos(as) que tiveram pouca $(\mathrm{OR}=2,24)$, moderada $(\mathrm{OR}=4,19)$ ou muita $(\mathrm{OR}=6,63)$ dificuldade com as atividades remotas, nos(as) que possuíam computador com uso compartilhado $(\mathrm{OR}=1,16)$, nos(as) que consumiam bebida alcoólica $(\mathrm{OR}=1,29)$, nos(as) que não estavam praticando atividade física $(\mathrm{OR}=1,12), \operatorname{nos}(\mathrm{as})$ que tiveram sua atividade de lazer diminuída $(\mathrm{OR}=1,21)$ e nos(as) que não estavam realizando atividade de lazer $(\mathrm{OR}=1,47)$. Como fatores de proteção encontram-se os homens $(\mathrm{OR}=0,86)$, os(as) com idade de 41 a 60 anos $(\mathrm{OR}=0,8)$ e mais de 60 anos $(\mathrm{OR}=0,62)$, os(as) que exerciam trabalho remunerado em outra instituição $(\mathrm{OR}=0,86)$ e os(as) que estavam contratados(as) ou designados(as) $(\mathrm{OR}=0,83)$.

Em relação aos que estavam insatisfeitos(as), quando comparados(as) aos(as) satisfeitos(as), as chances foram maiores nos(as) professores(as) que viviam sem cônjuge $(\mathrm{OR}=1,23)$, nos(as) que possuíam 21 ou mais anos de docên- 
Tabela 1. Análise descritiva e bivariada da satisfação com o trabalho docente, segundo perfil sociodemográfico e econômico, perfil ocupacional e comportamentos/hábitos durante a pandemia entre professores(as). Projeto ProfSMoc - Etapa Minas Covid, 2020 (n=15.641).

\begin{tabular}{|c|c|c|c|c|c|}
\hline Variáveis & $\mathbf{n}(\%)$ & $\begin{array}{c}\text { Satisfeito(a) } \\
\text { n (\%) }\end{array}$ & $\begin{array}{c}\text { Indiferente } \\
\mathbf{n}(\%)\end{array}$ & $\begin{array}{c}\text { Insatisfeito(a) } \\
\mathbf{n}(\%)\end{array}$ & $\mathbf{p}^{\star}$ \\
\hline \multicolumn{6}{|c|}{ Perfil sociodemográfico e econômico } \\
\hline \multicolumn{6}{|c|}{ Sexo } \\
\hline Feminino & $12.817(81,9)$ & $2.728(21,3)$ & $5.866(45,8)$ & $4.223(32,9)$ & 0,000 \\
\hline Masculino & $2.824(18,1)$ & $647(22,9)$ & $1.129(40,0)$ & $1.048(37,1)$ & \\
\hline \multicolumn{6}{|l|}{ Idade } \\
\hline De 21 a 40 anos & $6.447(41,2)$ & $1.321(20,5)$ & $2.900(45,0)$ & $2.226(34,5)$ & 0,000 \\
\hline De 41 a 60 anos & $8.793(56,2)$ & $1.937(22,0)$ & $3.922(44,6)$ & $2.934(33,4)$ & \\
\hline Mais de 60 anos & $401(2,6)$ & $117(29,2)$ & $173(43,1)$ & $111(27,7)$ & \\
\hline \multicolumn{6}{|l|}{ Área censitária } \\
\hline Urbana & $13.565(86,7)$ & $2.939(21,7)$ & $5.992(44,2)$ & $4.634(34,2)$ & 0,001 \\
\hline Rural & $2.076(13,3)$ & $436(21,0)$ & $1.002(48,3)$ & $637(30,7)$ & \\
\hline \multicolumn{6}{|l|}{ Renda familiar } \\
\hline De 1 a 2 salários & $3.969(25,4)$ & $86(20,2)$ & $176(41,3)$ & $164(38,5)$ & 0,000 \\
\hline De 3 a 5 salários & $9.301(59,5)$ & $377(19,4)$ & $825(42,4)$ & $743(38,2)$ & \\
\hline De 6 a 9 salários & $1.945(12,4)$ & $1.956(21,0)$ & $4.191(45,1)$ & $3.154(33,9)$ & \\
\hline 10 ou mais salários & $426(2,7)$ & $956(24,1)$ & $1.803(45,4)$ & $1.210(30,5)$ & \\
\hline \multicolumn{6}{|l|}{ Alteração na renda familiar } \\
\hline Aumentou & $304(1,9)$ & $68(22,4)$ & $124(40,8)$ & $112(36,8)$ & 0,000 \\
\hline Foi mantida igual & $8.947(57,2)$ & $2.188(24,5)$ & $3.978(44,5)$ & $2.781(31,1)$ & \\
\hline Diminuiu & $6.390(40,9)$ & $1.119(17,5)$ & $2.893(45,3)$ & $2.378(37,2)$ & \\
\hline \multicolumn{6}{|l|}{ Outro trabalho remunerado } \\
\hline Não & $10.269(65,7)$ & $2.200(21,4)$ & $4.661(45,4)$ & $3.408(33,2)$ & 0,001 \\
\hline Sim, em outra instituição & $3.815(24,3)$ & $874(22,9)$ & $1.669(43,7)$ & $1.272(33,3)$ & \\
\hline Sim, fora da docência & $1.557(10,0)$ & $301(19,3)$ & $665(42,7)$ & $591(38,0)$ & \\
\hline \multicolumn{6}{|l|}{ Situação conjugal } \\
\hline Com cônjuge & $10.453(66,8)$ & $2.293(21,9)$ & $4.716(45,1)$ & $3.444(32,9)$ & 0,016 \\
\hline Sem cônjuge & $5.188(33,2)$ & $1.082(20,9)$ & $2.279(43,9)$ & $1.827(35,2)$ & \\
\hline \multicolumn{6}{|l|}{ Filho(s) } \\
\hline Sim & $11.350(72,6)$ & $2.439(21,5)$ & $5.157(45,4)$ & $3.754(33,1)$ & 0,008 \\
\hline Não & $4.291(27,4)$ & $936(21,8)$ & $1.838(42,8)$ & $1.517(35,4)$ & \\
\hline
\end{tabular}

continua

cia $(\mathrm{OR}=1,19)$, $\operatorname{nos}($ as $)$ que apresentaram pouca $(\mathrm{OR}=2,03)$, moderada $(\mathrm{OR}=7,00)$ ou muita $(\mathrm{OR}=37,60)$ dificuldade com as atividades remotas, nos(as) que possuíam computador com uso compartilhado $(\mathrm{OR}=1,15)$ e nos(as) que não tinham computador $(\mathrm{OR}=1,40)$, nos(as) tabagistas $(\mathrm{OR}=1,27)$, nos(as) que consumiam bebida alcoólica $(\mathrm{OR}=1,54)$, nos(as) que não estavam praticando atividade física $(\mathrm{OR}=1,22), \operatorname{nos}($ as $)$ que tiveram sua atividade de lazer diminuída $(\mathrm{OR}=1,33)$ e nos(as) que não estavam realizando atividade de lazer $(\mathrm{OR}=1,49)$. Como fatores de proteção encontraram-se os(as) professores(as) com idade de 41 a 60 anos $(\mathrm{OR}=0,77)$ e mais de 60 anos $(\mathrm{OR}=0,51)$, os(as) que estavam contra- tados(as) ou designados(as) $(\mathrm{OR}=0,59)$, os(as) que não possuíam pós-graduação $(\mathrm{OR}=0,62)$ e os(as) que tinham especialização $(\mathrm{OR}=0,47) \mathrm{e}$, entre os(as) que não alteraram as atividades de lazer $(\mathrm{OR}=0,76)$.

\section{Discussão}

Os dados do presente estudo evidenciaram que aproximadamente metade dos(as) professores(as) investigados(as) se sentiam indiferentes em relação à satisfação com o trabalho docente durante a pandemia e um terço deles(as) estavam insatisfeitos(as). A indiferença sugere um estágio 
Tabela 1. Análise descritiva e bivariada da satisfação com o trabalho docente, segundo perfil sociodemográfico e econômico, perfil ocupacional e comportamentos/hábitos durante a pandemia entre professores(as). Projeto ProfSMoc - Etapa Minas Covid, 2020 ( $\mathrm{n}=15.641)$.

\begin{tabular}{|c|c|c|c|c|c|}
\hline \multirow{2}{*}{ Variáveis } & \multirow{2}{*}{$\mathbf{n}(\%)$} & \multirow{2}{*}{$\begin{array}{c}\text { Satisfeito(a) } \\
\text { n (\%) }\end{array}$} & \multirow{2}{*}{$\begin{array}{c}\text { Indiferente } \\
\mathbf{n}(\%)\end{array}$} & \multirow{2}{*}{$\frac{\text { Insatisfeito(a) }}{\mathbf{n}(\%)}$} & \multirow{2}{*}{$\mathbf{p}^{*}$} \\
\hline & & & & & \\
\hline \multicolumn{6}{|l|}{ Perfil ocupacional } \\
\hline \multicolumn{6}{|l|}{ Anos de docência } \\
\hline De 1 a 10 anos & $5.941(38,0)$ & $1.358(22,9)$ & $2.608(43,9)$ & $1.975(33,2)$ & 0,004 \\
\hline De 11 a 20 anos & $5.788(37,0)$ & $1.240(21,4)$ & $2.633(45,5)$ & $1.915(33,1)$ & \\
\hline 21 ou mais anos & $3.911(25,0)$ & $777(19,9)$ & $1.754(44,8)$ & $1.380(35,3)$ & \\
\hline \multicolumn{6}{|l|}{ Horas semanais de trabalho docente } \\
\hline Até 19 horas & $3.613(23,1)$ & $813(22,5)$ & $1.587(43,9)$ & $1.214(33,6)$ & 0,003 \\
\hline De 20 a 39 horas & $9.554(61,1)$ & $2.058(21,5)$ & $4.349(45,5)$ & $3.147(32,9)$ & \\
\hline 40 horas ou mais & $2.472(15,8)$ & $504(20,4)$ & $1.058(42,8)$ & $910(36,8)$ & \\
\hline \multicolumn{6}{|l|}{ Vínculo com a escola } \\
\hline Concursado(a)/efetivo(a) & $8.440(54,0)$ & $1.574(18,6)$ & $3.635(43,1)$ & $3.231(38,3)$ & 0,000 \\
\hline Contratado(a)/designado(a) & $7.201(46,0)$ & $1.801(25,0)$ & $3.360(46,7)$ & $2.040(28,3)$ & \\
\hline \multicolumn{6}{|l|}{ Possui pós-graduação } \\
\hline Mestrado e/ou doutorado & $692(4,4)$ & $112(16,2)$ & $251(36,3)$ & $329(47,2)$ & 0,000 \\
\hline Especialização & $11.115(71,1)$ & $2.522(22,7)$ & $5.041(45,2)$ & $3.552(32,0)$ & \\
\hline Não & $3.834(24,5)$ & $741(19,3)$ & $1.703(44,4)$ & $1.390(36,3)$ & \\
\hline \multicolumn{6}{|c|}{$\begin{array}{l}\text { Realização de trabalho remoto durante } \\
\text { a pandemia }\end{array}$} \\
\hline Sim & $15.520(99,2)$ & $3.351(21,6)$ & $6.955(44,8)$ & $5.214(33,6)$ & 0,006 \\
\hline Não & $121(0,8)$ & $24(19,8)$ & $40(33,1)$ & $57(47,1)$ & \\
\hline \multicolumn{6}{|c|}{ Dificuldades com atividades remotas } \\
\hline Nenhuma dificuldade & $1.472(9,4)$ & $768(52,2)$ & $510(34,6)$ & $194(13,2)$ & 0,000 \\
\hline Pouca & $4.184(26,8)$ & $1.313(31,4)$ & $2.122(50,7)$ & $749(17,9)$ & \\
\hline Moderada & $6.777(43,3)$ & $1.108(16,3)$ & $3.435(50,7)$ & $2.234(33,0)$ & \\
\hline Muita & $3.208(20,5)$ & $186(5,8)$ & $928(28,9)$ & $2.094(65,3)$ & \\
\hline \multicolumn{6}{|l|}{ Possui computador } \\
\hline Sim, de uso pessoal & $7.757(49,6)$ & $1.926(24,8)$ & $3.412(44,0)$ & $2.419(31,2)$ & 0,000 \\
\hline Sim, compartilhado & $7.310(46,7)$ & $1.350(18,5)$ & $3.371(46,1)$ & $2.589(35,4)$ & \\
\hline Não & $574(3,7)$ & $99(17,2)$ & $212(36,9)$ & $264(45,8)$ & \\
\hline \multicolumn{6}{|c|}{$\begin{array}{l}\text { Comportamentos/hábitos durante a } \\
\text { pandemia }\end{array}$} \\
\hline \multicolumn{6}{|l|}{ Tabagista } \\
\hline Não & $14.123(90,3)$ & $3.082(21,8)$ & $6.382(45,2)$ & $4.659(33,0)$ & 0,000 \\
\hline Ex-fumante & $665(4,2)$ & $130(19,5)$ & $292(43,9)$ & $243(36,5)$ & \\
\hline Sim & $853(5,5)$ & $163(19,1)$ & $321(37,6)$ & $369(43,3)$ & \\
\hline \multicolumn{6}{|l|}{ Consumo de bebida alcoólica } \\
\hline Não & $9.290(59,4)$ & $2.258(24,3)$ & $4.221(45,4)$ & $2.811(30,3)$ & 0,000 \\
\hline Sim & $6.351(40,6)$ & $1.117(17,6)$ & $2.774(43,7)$ & $2.460(38,7)$ & \\
\hline \multicolumn{6}{|l|}{ Prática de atividade física } \\
\hline Sim & $8798(56,2)$ & $2.045(23,2)$ & $3.943(44,8)$ & $2.810(31,9)$ & 0,000 \\
\hline Não & $6843(43,8)$ & $1.330(19,4)$ & $3.052(44,6)$ & $2.461(36,0)$ & \\
\hline \multicolumn{6}{|l|}{ Atividade de lazer } \\
\hline Aumentou & $876(5,6)$ & $238(27,2)$ & $372(42,5)$ & $266(30,4)$ & 0,000 \\
\hline Não se alterou & $3.005(19,2)$ & $920(30,6)$ & $1.345(44,8)$ & $740(24,6)$ & \\
\hline Diminuiu & $5.033(32,2)$ & $1.031(20,5)$ & $2.184(43,4)$ & $1.818(36,1)$ & \\
\hline Não está realizando & $6.727(43,0)$ & $1.186(17,6)$ & $3.094(46,0)$ & $2.447(36,4)$ & \\
\hline
\end{tabular}

*Variáveis selecionadas para compor inicialmente o modelo múltiplo, com nível descritivo inferior ou igual a $20 \%$ (p $\leq 0,20)$. 
intermediário entre os sentimentos de satisfação e insatisfação, trazendo em si uma ideia de gradação quanto aos sentimentos negativos relacionados ao trabalho. Assim, observou-se que quase $80 \% \operatorname{dos}$ (as) professores(as) investigados(as) não se encontravam satisfeitos(as) com o trabalho durante a pandemia.

O trabalho remoto em decorrência da pandemia da COVID-19 não inaugurou a situação de intensificação do trabalho docente $e^{8,13,14}$, no entanto trouxe ainda mais sobrecarga de trabalho para os(as) professores(as) ${ }^{3}$, com transformação das abordagens pedagógicas em encontros virtuais, da sala de casa em sala de aula, além da necessidade da aprendizagem de novas formas de ensinar ${ }^{4}$. Situações capazes de ocasionar maior insatisfação com o trabalho entre os(as) professores(as).

Entre os fatores associados à indiferença e à insatisfação, destacam-se, para cada uma, dois grupos de resultados: as variáveis que apresentaram associação positiva e aquelas que se comportaram como fatores de proteção.
Maiores chances de indiferença e insatisfação com o trabalho foram observadas entre os(as) professores(as) que viviam sem cônjuge, aqueles(as) que atuavam na docência há 21 anos ou mais, os(as) que enfrentavam algum grau de dificuldade com as atividades remotas, compartilhavam o computador com alguém em casa, aqueles(as) que consumiam bebidas alcoólicas, não praticavam atividades físicas e entre os(as) que não estavam realizando atividades de lazer durante a pandemia ou reduziram o tempo destinado a elas.

Os(as) professores(as) que viviam sem cônjuge apresentaram maiores chances de indiferença e insatisfação em relação ao trabalho. Estudo anterior mostrou que a experiência de viver com companheiro está associada de forma positiva ao crescimento profissional dos indivíduos ${ }^{15}$. O cenário de distanciamento social, propiciado pela pandemia, por si só, apresenta impactos na saúde mental das pessoas ${ }^{16}$. Tal fato, associado à perda do relacionamento presencial que envolve a docência, poderia explicar o impacto na satisfa-

Tabela 2. Regressão Logística Multinomial, tendo a satisfação com o trabalho docente como categoria de referência. Projeto ProfSMoc - Etapa Minas Covid, 2020 (n=15.641).

\begin{tabular}{|c|c|c|c|c|}
\hline \multirow{2}{*}{ Variáveis } & \multirow{2}{*}{$\begin{array}{c}\text { Indiferente } \\
\text { OR (IC95\%) }\end{array}$} & \multirow{2}{*}{$\mathbf{p}$} & \multirow{2}{*}{$\begin{array}{c}\text { Insatisfeito(a) } \\
\text { OR (IC95\%) }\end{array}$} & \multirow{2}{*}{$\mathbf{p}$} \\
\hline & & & & \\
\hline \multicolumn{5}{|c|}{ Perfil Sociodemográfico e econômico } \\
\hline \multicolumn{5}{|c|}{ Sexo } \\
\hline Feminino & 1 & & 1 & \\
\hline Masculino & $0,86(0,76-0,96)$ & 0,012 & $1,01(0,89-1,15)$ & 0,800 \\
\hline \multicolumn{5}{|l|}{ Idade } \\
\hline De 21 a 40 anos & 1 & & 1 & \\
\hline De 41 a 60 anos & $0,80(0,72-0,89)$ & 0,000 & $0,77(0,69-0,87)$ & 0,000 \\
\hline Mais de 60 anos & $0,62(0,47-0,81)$ & 0,001 & $0,51(0,37-0,70)$ & 0,000 \\
\hline \multicolumn{5}{|l|}{ Área censitária } \\
\hline Urbana & 1 & & 1 & \\
\hline Rural & $1,18(1,04-1,35)$ & 0,009 & $1,02(0,88-1,18)$ & 0,773 \\
\hline \multicolumn{5}{|l|}{ Alteração na renda familiar } \\
\hline Aumentou & 1 & & 1 & \\
\hline Foi mantida igual & $1,01(0,74-1,38)$ & 0,938 & $0,76(0,54-1,06)$ & 0,115 \\
\hline Diminuiu & $1,41(1,03-1,93)$ & 0,032 & $1,24(0,88-1,75)$ & 0,202 \\
\hline \multicolumn{5}{|l|}{ Outro trabalho remunerado } \\
\hline Não & 1 & & 1 & \\
\hline Sim, em outra instituição & $0,86(0,78-0,95)$ & 0,005 & $0,90(0,80-1,01)$ & 0,074 \\
\hline Sim, fora da docência & $1,06(0,91-1,24)$ & 0,420 & $1,17(0,99-1,38)$ & 0,065 \\
\hline \multicolumn{5}{|l|}{ Situação conjugal } \\
\hline Com cônjuge & 1 & & 1 & \\
\hline Sem cônjuge & $1,13(1,02-1,24)$ & 0,011 & $1,23(1,11-1,37)$ & 0,000 \\
\hline
\end{tabular}


Tabela 2. Regressão Logística Multinomial, tendo a satisfação com o trabalho docente como categoria de referência. Projeto ProfSMoc - Etapa Minas Covid, 2020 (n=15.641).

\begin{tabular}{|c|c|c|c|c|}
\hline \multirow{2}{*}{ Variáveis } & \multirow{2}{*}{$\begin{array}{c}\text { Indiferente } \\
\text { OR (IC95\%) }\end{array}$} & \multirow{2}{*}{$\mathbf{p}$} & \multirow{2}{*}{$\begin{array}{c}\text { Insatisfeito(a) } \\
\text { OR (IC95\%) }\end{array}$} & \multirow{2}{*}{$\mathbf{p}$} \\
\hline & & & & \\
\hline \multicolumn{5}{|l|}{ Perfil ocupacional } \\
\hline \multicolumn{5}{|l|}{ Anos de docência } \\
\hline De 1 a 10 anos & 1 & & 1 & \\
\hline De 11 a 20 anos & $1,16(1,12-1,47)$ & 0,005 & $1,04(0,93-1,18)$ & 0,440 \\
\hline 21 ou mais anos & $1,28(1,04-1,29)$ & 0,000 & $1,19(1,02-1,39)$ & 0,022 \\
\hline \multicolumn{5}{|l|}{ Vínculo com a escola } \\
\hline Concursado(a)/efetivo(a) & 1 & & 1 & \\
\hline Contratado(a)/designado(a) & $0,83(0,76-0,91)$ & 0,000 & $0,59(0,53-0,65)$ & 0,000 \\
\hline \multicolumn{5}{|l|}{ Possui pós-graduação } \\
\hline Mestrado e/ou doutorado & 1 & & 1 & \\
\hline Especialização & $0,79(0,62-1,01)$ & 0,063 & $0,47(0,36-0,60)$ & 0,000 \\
\hline Não & $0,93(0,72-1,19)$ & 0,581 & $0,62(0,48-0,81)$ & 0,000 \\
\hline \multicolumn{5}{|l|}{ Dificuldades com atividades remotas } \\
\hline Nenhuma dificuldade & 1 & & 1 & \\
\hline Pouca & $2,24(1,96-2,56)$ & 0,000 & $2,03(1,69-2,45)$ & 0,000 \\
\hline Moderada & $4,19(3,66-4,79)$ & 0,000 & $7,00(5,85-8,37)$ & 0,000 \\
\hline Muita & $6,63(5,36-7,95)$ & 0,000 & $37,60(30,07-47,01)$ & 0,000 \\
\hline \multicolumn{5}{|l|}{ Possui computador } \\
\hline Sim, de uso pessoal & 1 & & 1 & \\
\hline Sim, compartilhado & $1,16(1,06-1,27)$ & 0,001 & $1,15(1,04-1,28)$ & 0,006 \\
\hline Não & $0,97(0,75-1,25)$ & 0,835 & $1,40(1,06-1,83)$ & 0,015 \\
\hline \multicolumn{5}{|c|}{ Comportamentos/hábitos durante a pandemia } \\
\hline \multicolumn{5}{|c|}{ Tabagista } \\
\hline Não & 1 & & 1 & \\
\hline Ex-fumante & $1,04(0,83-1,30)$ & 0,694 & $1,06(0,83-1,35)$ & 0,621 \\
\hline Sim & $0,90(0,73-1,10)$ & 0,325 & $1,27(1,03-1,58)$ & 0,026 \\
\hline \multicolumn{5}{|l|}{ Consumo de bebida alcoólica } \\
\hline Não & 1 & & 1 & \\
\hline Sim & $1,29(1,18-1,42)$ & 0,000 & $1,54(1,39-1,71)$ & 0,000 \\
\hline \multicolumn{5}{|l|}{ Prática de atividade física } \\
\hline Sim & 1 & & 1 & \\
\hline Não & $1,12(1,03-1,23)$ & 0,007 & $1,22(1,10-1,34)$ & 0,000 \\
\hline \multicolumn{5}{|l|}{ Atividade de lazer } \\
\hline Aumentou & 1 & & 1 & \\
\hline Não se alterou & $0,97(0,80-1,18)$ & 0,787 & $0,76(0,61-0,95)$ & 0,018 \\
\hline Diminuiu & $1,21(1,01-1,46)$ & 0,043 & $1,33(1,08-1,64)$ & 0,008 \\
\hline Não está realizando & $1,47(1,22-1,77)$ & 0,000 & $1,49(1,20-1,84)$ & 0,000 \\
\hline
\end{tabular}

Fonte: Elaborado pelas autoras.

ção dos(as) professores(as), especialmente entre aqueles(as) que não possuem um(a) companheiro(a), por intensificar o sentimento de solidão proporcionado pelo distanciamento social.

Os(as) professores(as) que possuíam 21 anos ou mais de atuação na docência apresentaram maiores chances tanto de indiferença quanto de insatisfação com o trabalho. Possível explicação pode ser o fato de que o(a) profissional, já cansado(a) pelos anos de trabalho, ainda precisou se adaptar a uma nova modalidade de ensino imposta pelo distanciamento, o que implicaria em novos desafios laborais. Estudo entre professores da rede municipal de Vitória da Conquista, na 
Bahia, evidenciou que um maior tempo de docência está associado a maiores prevalências de cansaço mental e de nervosismo, fatores esses, que contribuem para a insatisfação profissional ${ }^{17}$. Os resultados do presente estudo mostraram, ainda, maior chance de indiferença entre os(as) professores(as) que atuavam na profissão entre 11 e 20 anos.

Observou-se que maiores chances de indiferença e também de insatisfação em relação ao trabalho estiveram associadas a enfrentar algum grau de dificuldade com as atividades remotas desenvolvidas durante a pandemia. Estudo com professores indonésios mostrou que para muitos deles o uso de tecnologias tem sido um exercício árduo, que causa ansiedade nessa fase de adaptação durante a pandemia ${ }^{5}$. Habituados com as práticas tradicionais de ensino que incluem o uso de quadro, pincel e projetor de slides, os(as) professores(as) encontram-se diante do desafio de preparar e apresentar diferentes temáticas com o uso de tecnologias digitais de comunicação e informação $0^{4,5}$.

Em relação a possuir computador, os resultados evidenciaram que maiores chances de indiferença e insatisfação estiveram associadas ao uso compartilhado do equipamento e, ainda maior insatisfação ocorreu entre os(as) professores(as) que não tinham computador. $\mathrm{O}(\mathrm{a})$ professor(a) foi responsável por adequar o ambiente domiciliar para o trabalho remoto ${ }^{4}$, adequação essa que prevê a necessidade de equipamentos e acessórios. Pode-se esperar prejuízo nas atividades docentes no cenário de ausência do equipamento ou, ainda, quando o computador precisava ser compartilhado, por exemplo, com um filho em idade escolar, também em ensino remoto.

No presente estudo, o consumo de bebida alcoólica esteve significativamente associado à indiferença e à insatisfação com o trabalho. Ainda, o tabagismo mostrou-se associado à maior chance de insatisfação com o trabalho. O álcool e o tabaco figuram entre as substâncias psicoativas lícitas mais utilizadas, com frequência, associados a situações de ansiedade e estresse $\mathrm{e}^{18}$. Considerando as circunstâncias sobre as quais os(as) professores(as) usualmente estão submetidos, tais como a intensa exigência cognitiva, jornadas que ultrapassam 40 horas semanais, ministrar aulas, pesquisar, participar de reuniões deliberativas e orientar estudantes, comportamentos como o consumo de álcool e tabaco seriam buscados como mecanismos de fuga às tensões vivenciadas $^{18}$. O contexto da pandemia da COVID-19 exigiu maior disponibilidade de tempo dos(as) professores(as), contribuindo com níveis mais elevados de estresse ${ }^{3}$, favorecendo o consumo de substâncias e sentimentos de insatisfação em relação ao trabalho realizado.

Os resultados obtidos neste estudo mostraram associações tanto entre a indiferença quanto a insatisfação com o trabalho e a ausência de prática de atividades físicas, bem como com a redução e ausência de atividades de lazer. Atividades realizadas fora do trabalho têm potencial efeito na recuperação de tensões acumuladas durante a realização das tarefas profissionais e a redução do tempo dedicado a elas pode produzir efeitos negativos sobre a saúde física e mental ${ }^{19}$ e possíveis repercussões sobre a satisfação com o trabalho. $\mathrm{O}$ que corrobora com o resultado observado de menor insatisfação com o trabalho entre os(as) professores(as) que relataram não ter havido alteração no tempo destinado às atividades de lazer.

Os(as) professores(as) que atuavam em escolas localizadas na zona rural apresentaram maior chance de indiferença em relação à satisfação com o trabalho. Tal achado pode representar um sentimento de resiliência frente às dificuldades enfrentadas pelos(as) professores(as) e ser justificado pelas precárias condições de trabalho das escolas rurais, as quais possuem um acervo muito modesto de recursos bibliográficos e audiovisuais $^{20}$, o que pode ter dificultado a realização do ensino remoto. Estudo conduzido em escolas rurais do estado de Goiás evidenciou que os desafios em relação ao ensino enfrentados durante a pandemia são reflexos não só da falta de acesso ao sinal de internet, mas também da falta de acompanhamento da família no que tange as atividades escolares ${ }^{21}$. Embora essa falta de acompanhamento da família não seja uma realidade exclusiva do contexto rural, o estudo demonstra que, mesmo antes da pandemia, as famílias no campo já apresentavam dificuldades para acompanhar a aprendizagem das crianças, e a adesão ao ensino remoto conferiu ainda maiores dificuldades $^{21}$. Os desafios impostos pela pandemia aos(as) professores(as) da zona rural podem potencializar sentimentos negativos relacionados ao trabalho. São necessários novos estudos que avaliem a relação entre a família e a escola nos diferentes contextos urbano e rural.

Outra variável associada somente à maior chance de indiferença foi a redução da renda familiar durante a pandemia. A remuneração da profissão docente é um dos aspectos que pode gerar satisfação profissional ou, em contrapartida, gerar insatisfação ${ }^{22}$. Além disso, pode-se esperar que a redução da renda em função do 
desemprego de um dos membros da família em decorrência da pandemia, por exemplo, atuaria como fator de frustração e insatisfação.

Maior idade e ser contratado(a)/designado(a) atuaram como fatores de proteção no presente estudo tanto para a indiferença quanto para a insatisfação em relação ao trabalho. Os(as) professores(as) com idade superior a 41 anos apresentaram menor chance de indiferença e insatisfação quando comparados àqueles que tinham entre 21 e 40 anos. Estudo português evidenciou uma relação diretamente proporcional entre a idade e a satisfação profissional, de forma que quanto maior a idade, mais satisfeito(a) o(a) professor(a) se sente em relação ao trabalho. Os(as) professores(as) de idade mais avançada, em geral, estão mais envolvidos com a profissão, haja vista que, na maioria das vezes, esses(as) docentes são efetivos(as) e encontram maior suporte relacional no ambiente escolar, além de possuírem uma reputação já estabelecida e uma valorização por parte da escola ${ }^{23}$.

Em relação ao vínculo com a escola, os(as) professores(as) contratados(as) ou designados(as) também apresentaram menores chances tanto de indiferença quanto de insatisfação com o trabalho. Resultado divergente ao encontrado em estudo realizado previamente à pandemia com professores(as) do estado do Paraná que evidenciou que, entre os aspectos que geram insatisfação no trabalho, está a situação de serem temporários. O que obriga que os(as) docentes trabalhem em várias escolas, atendam a diferentes demandas e aceitem as aulas disponíveis em estabelecimentos diferentes, se deslocando, às vezes num único turno, entre escolas distantes ${ }^{24}$.

Os resultados evidenciaram que os professores do sexo masculino apresentam menor chance de indiferença em relação à satisfação com o trabalho. Um estudo longitudinal feito com professores egípcios do ensino básico avaliou a satisfação desses profissionais em relação ao aumento salarial, evidenciando que, após esse aumento, os professores avaliaram sua satisfação no trabalho significativamente mais alta do que as professoras $^{25}$. Uma revisão sobre satisfação dos docentes do ensino superior apontou que, em alguns estudos, as professoras apresentavam níveis mais elevados de satisfação do que os professores. Contudo, ressalta ainda que há estudos que afirmam não haver relação entre o sexo e a satisfação profissional dos professores ${ }^{26}$. Possível explicação para a diferença de satisfação entre professores e professoras seria o fato de as mulheres apresentarem, com frequência, dupla ou tripla jornada de trabalho, de forma a acumularem afazeres devido à necessidade de conciliar as atividades domésticas, familiares e profissionais ${ }^{27,28}$. Durante a pandemia, devido à transferência da sala de aula para casa, a conciliação das tarefas extra laborais e laborais tornou-se ainda mais desafiadora, o que pode explicar o menor impacto nos professores do sexo masculino em relação à satisfação com o trabalho, uma vez que as atividades domésticas são culturalmente, na maioria das vezes, atribuições das mulheres. Há que se destacar a predominância da população feminina entre os professores, característica majoritária nesse campo em âmbito nacional.

Os(as) professores(as) que relataram possuir outro trabalho remunerado em outra instituição escolar apresentaram menor chance de indiferença em relação à satisfação com o trabalho. Em geral, múltiplos vínculos de emprego são indicados como fatores de sobrecarga ocupacional, com riscos à saúde do(a) trabalhador (a $)^{14}$.

Em comparação com os(as) professores(as) que possuíam mestrado e/ou doutorado, aqueles(as) que possuíam especialização e também aqueles(as) que não haviam feito pós-graduação apresentaram menor chance de insatisfação com o trabalho. Entende-se que a formação permanente é elemento necessário para a atividade docente, conferindo maior grau de capacitação ${ }^{29}$, o que poderia repercutir nos níveis de satisfação com o trabalho. O maior nível de formação constitui elemento de insatisfação frente a condições de trabalho precárias e salários defasados, situações frequentes para a categoria profissional investigada ${ }^{8,14}$, adicionados aos desafios impostos pelo processo de trabalho durante a pandemia.

Algumas limitações precisam ser consideradas. Websurveys ${ }^{11}$ apresentam possibilidade de viés de seleção, pois dependem do acesso à internet. Além disso, as respostas são baseadas no autorrelato. Porém, esse tipo de pesquisa também apresenta vantagens, como a possibilidade de realização de coletas de dados a distância (principalmente durante um momento de pandemia, em que é extremamente importante manter o distanciamento social), atingir maior contingente populacional, alcançar grande abrangência geográfica, rapidez no planejamento e publicação dos resultados ${ }^{11}$. Outros pontos fortes do estudo são o rigor metodológico, a robustez da amostra, o apoio da SEE-MG, a boa distribuição da amostra pelo estado, a representatividade de $93,2 \%$ dos municípios mineiros e cobertura de 13,3\% de professores(as) atuantes na zona rural. 
Aproximadamente $80 \%$ dos(as) professores(as) do presente estudo não estavam satisfeitos(as) com o trabalho docente durante a pandemia. Essa "não satisfação" apresenta relação com a intensificação das atribuições relacionadas à docência e as mudanças causadas no sistema educacional diante da pandemia da COVID-19.

O presente estudo observou que as chances de estarem insatisfeitos foram maiores entre os(as) professores(as) que viviam sem cônjuge, aqueles(as) com maior tempo de docência, que apresentaram dificuldade com as aulas remotas, que não possuíam computador ou tinham o uso do mesmo compartilhado, os(as) tabagistas, os(as) que consumiam bebidas alcóolicas, aqueles(as) que não praticaram atividade física e os(as) que estavam com atividades de lazer diminuída e ausente durante a pandemia.

Desta forma, o presente estudo indica que as mudanças causadas no sistema educacional provocada pela pandemia da COVID-19, impactou diretamente a rotina do trabalho do(a) professor(a), causando prejuízos aos mesmos e contribuindo para a insatisfação com o trabalho dos(as) professores(as) neste período. As políticas públicas e todas as esferas de gestão da educação devem se remodelar para este contexto, a fim de possibilitar que os(as) docentes trabalhem de maneira satisfatória, propiciando um ensino de qualidade e protegendo a saúde deste trabalhador(a).

Os resultados obtidos nesta investigação têm o potencial de contribuir para a discussão mais ampla e atual sobre o assunto, com base na produção de conhecimentos relevantes no campo da saúde do(a) trabalhador(a) da área da docência, mediante as dificuldades e escassez de estudos de tal natureza, escopo e amplitude com professores(as). Medidas de vigilância sobre o trabalho e a saúde docente devem ser adotadas, especialmente no contexto da pandemia. $\mathrm{O}$ monitoramento contínuo da saúde dos(as) profissionais da educação precisa ser direcionado sob o enfoque da saúde coletiva e da vigilância em saúde do(as) trabalhador(as), e configura-se como importante procedimento de proteção à vida destes(as) profissionais. Programas que trabalhem com adoecimento docente no âmbito das políticas públicas são necessários, na perspectiva da articulação entre as políticas de saúde e de educação no país.

\section{Colaboradores}

RRV Silva contribuiu na concepção do projeto, coleta de dados, interpretação dos dados, redação e revisão final. REC Barbosa contribuiu na concepção do projeto, coleta de dados, redação e revisão final. NSS Silva contribuiu na concepção do projeto, coleta e organização dos dados, análise estatística, interpretação dos dados, redação e revisão final do artigo. L Pinho contribuiu na concepção do projeto, coleta de dados da pesquisa e revisão final do artigo. TB Ferreira contribuiu na interpretação dos dados e na redação. BB Moreira contribuiu na interpretação dos dados e na redação. MFSF Brito contribuiu na concepção do projeto, interpretação dos dados, redação e revisão final. DS Haikal contribuiu na concepção do projeto, coleta e organização dos dados, redação e revisão final do artigo.

\section{Agradecimentos}

Agradecemos os Professores do estado de Minas Gerais por participarem do Projeto ProfSMoc Etapa Minas Covid, ao apoio da Unimontes e da SEE-MG e à Coordenação de Aperfeiçoamento de Pessoal de Nível Superior (CAPES) pela concessão de Bolsas. 


\section{Referências}

1. Minas Gerais. Deliberação do Comitê Extraordinário COVID-19 no 15, de 20 de março de 2020. Dispõe sobre a suspensão das atividades educacionais e dá outras providências. Belo Horizonte: Secretaria de Estado de Fazenda de Minas Gerais; 2020.

2. Agência Minas. Secretária de Educação detalha Regime de Estudo não Presencial na rede estadual de ensino [Internet]. 2020 [acessado 2021 abr 28]. Disponível em: http://agenciaminas.mg.gov.br/noticia/secretariade-educacao-detalha-regime-de-estudo-nao-presencial-na-rede-estadual-de-ensino.

3. Saraiva K, Traversini C, Lockmann K. A educação em tempos de COVID-19: ensino remoto e exaustão do docente. Prax Educ 2020; 15: 1-24.

4. Souza KR, Santos GB, Rodrigues MAS, Felix EG, Gomes L, Rocha GL, Conceição RCM, Rocha FS, Peixoto RB. Trabalho remoto, saúde docente e greve virtual em cenário de pandemia. Trab Educ Saude 2021; 19:e00309141.

5. Rasmitadila, Aliyyah RR, Rachmadtullah R, Samsudin A, Syaodih E, Nurtanto M, Tambunan ARS. The perceptions of primary school teachers of online learning during the COVID-19 pandemic period: a case study in Indonesia. J Ethn Cult Stu 2020; 7(2):90-109.

6. Wagner L, Carlesso JPP. Profissão docente: um estudo do abandono da carreira na contemporaneidade. Res Soc Develop 2019; 8(6):e386968.

7. Marqueze EC, Moreno CRC. Satisfação no trabalho: uma breve revisão. Rev Bras Saude Ocup 2005; 30(112):69-79.

8. Gasparini SM, Barreto SM, Assunção AA. O professor, as condições de trabalho e os efeitos sobre sua saúde. Educ Pesqui 2005; 31:189-199.

9. Secretaria de Estado de Educação de Minas Gerais. Relação de estabelecimentos de ensino ativos em Minas Gerais [Internet]. 2020 [acessado 2020 nov 02]. Disponível em: https://www2.educacao.mg.gov.br/parceiro/lista-de-escolas.

10. Boni RBD. Websurveys nos tempos de COVID-19. Cad Saude Publica 2020; 36(7):e00155820.

11. Eysenbach G. Improving the quality of Web surveys: the Checklist for Reporting Results of Internet E-Surveys (CHERRIES). J Med Internet Res 2004; 6(3):e34.

12. Instituto de Comunicação e Informação Científica e Tecnológica em Saúde (ICICT). Fundação Oswaldo Cruz (Fiocruz). ConVid - Pesquisa de Comportamentos. Maio, 2020 [Internet]. [acessado 2020 nov 02]. Disponível em: https://www.convid.fiocruz.br/.

13. Assunção AA, Oliveira DA. Intensificação do trabalho e saúde dos professores. Educ Soc 2009; 30:349-372.

14. Araújo TM, Pinho OS, Masson MLV. Trabalho e saúde de professoras e professores no Brasil: reflexões sobre trajetórias das investigações, avanços e desafios. Cad Saude Publica 2019; 35(Supl. 1):e00087318.

15. Gottman JM, Silver N. The seven principles for making marriage work: a practical guide from the country's foremost relationship expert. New York: Three River Press; 2007.

16. Lima RC. Distanciamento e isolamento sociais pela COVID-19 no Brasil: impactos na saúde mental. Physis 2020; 30(2):e300214.
17. Reis EJ, Araújo TM, Carvalho FM, Barbalho L, Silva MO. Docência e exaustão emocional. Educ Soc 2006; 27(94):229-253.

18. Franco LC, Monteiro PS. Padrão do consumo de álcool e tabaco entre os professores universitários. Rev Baiana Enferm 2016; 30(2):1-11.

19. Barbosa REC, Assunção AA, Araújo TM. Musculoskeletal pain among healthcare workers: an exploratory study on gender diferences. Am J Ind Med 2013; 56(10):1201-1212.

20. Ferreira LG. Professores da zona rural em início de carreira: narrativas de si e desenvolvimento professional [tese]. São Carlos: Universidade Federal de São Carlos; 2014.

21. Pereira AMF, Almeida MZCM. Escolas rurais de Rio Verde-GO: os desafios dos professores ao processo de ensino e aprendizagem em meio a pandemia. Human Tecnol 2020; 27(1):50-66.

22. Rabelo AO. A remuneração do professor é baixa ou alta? Uma contraposição de diferentes referenciais. Educ Rev 2010; 26(1):57-87.

23. Furtado SCO, Medeiros T. Satisfação profissional dos professores em pré-reforma. Rev Port Educ 2019; 32(2):24-39.

24. Larocca P, Girardi P. Trabalho, satisfação e motivação docente: um estudo exploratório com professores da educação básica. In: I Seminário Internacional de Representações Sociais, Subjetividade e Educação - SIRS$S E$. Curitiba: Pontifícia Universidade Católica do Paraná; 2011. p. 1932-1948.

25. Sabry M. Longitudinal effects of pay increase on teachers job satisfaction: a motivational perspective. J Int Soc Res 2010; 3(10):1-21.

26. Pocinho M, Fragoeiro JG. Satisfação dos docentes do ensino superior. Acta Colomb Psicol 2012; 15(1):87-97.

27. Marcacine PR, Castro SDS, Castro SSD, Meirelles MCCC, Haas VJ, Walsh IAPD. Qualidade de vida, fatores sociodemográficos e ocupacionais de mulheres trabalhadoras. Cien Saude Colet 2019; 24(3):749-760.

28. Gomes KK, Sanchez HM, Sanchez EGDM, Sbroggio Júnior AL, Arantes Filho WM, Silva LA, Barbosa MA, Porto CC. Qualidade de vida e qualidade de vida no trabalho em docentes da saúde de uma instituição de ensino superior. Rev Bras Med Trab 2017; 15(1):18-28.

29. Kamazaki SGC, Capellini VLMF, Oliveira AAS, Pedro KM. Formação de professores em educação especial na modalidade EaD: alguns apontamentos sobre seis cursos de especialização. EaD Foco 2017; 7(3):29-41.

Artigo apresentado em 25/01/2021

Aprovado em 09/09/2021

Versão final apresentada em 11/09/2021

Editores-chefes: Romeu Gomes, Antônio Augusto Moura da Silva 